\title{
Develop an efficient inoculation technique for Fusarium solani isolate "TJP-2178-10" pathogeny assessment in Phalaenopsis orchids
}

\author{
Shu-Yun Chen ${ }^{1}$, Yan-Jeng Wu' ${ }^{1}$, Ting-Fang Hsieh², Jiunn-Feng Sư ${ }^{2}$, Wei-Chiang Shen ${ }^{3}$, Yung-Hsiang Lai ${ }^{4}$, \\ Pen-Chih Lai ${ }^{4}$, Wen-Huei Chen ${ }^{5}$ and Hong-Hwa Chen ${ }^{1,5^{*}}$ (D)
}

\begin{abstract}
Background: Phalaenopsis is one of the important ornamental plants worldwide. It plays the most significant role in flower exportation in Taiwan. However, the yellow leaf disease caused by Fusarium spp. has reduced the orchid flower yield $10-50 \%$ yearly. Varieties resistant to yellow leaf disease associated with Fusarium is urgently needed for orchid growers and breeders, and is the ultimate solution for the long-term goal. To achieve this, phenotyping is the first step and the most necessary information for further studies, such as resistance gene identification, quantitative trait loci identification, and genome-wide association study.

Results: The inoculation of Fusarium was performed in either abbreviated stem or detached leaf, and the pros and cons were compared. The former is the general method of phenotyping for estimating the tolerance to yellow leaf disease of Phalaenopsis, but it is time-consuming and spacy, and thus not suitable for the assessment of large numbers of samples. In contrast, the latter not only showed a similar trend of disease severity with time reduced to only one fourth of the former one but also less space needed.
\end{abstract}

Conclusions: This solution allows a better phenotyping approach for the fast detection of yellow leaf disease associated with Fusarium in a large number of Phalaenopsis samples.

Keywords: Fusarium spp., Phalaenopsis, Phenotyping, Symptom, Yellow leaf disease

\section{Background}

Orchids are important flowers for horticultural production and exportation worldwide. Among them, Phalaenopsis app. are the most popular and significant orchids. In Taiwan, the export value of Phalaenopsis in 2019 was about 140 million US dollars which covered $75 \%$ of the total value of orchid exportation (https://www.coa.gov. tw/2.Fusarium spp., pathogens damage crop widely and cause up to $50 \%$ yield loss in soybean, banana, tomato, and wheat (Perincherry et al. 2019). For orchids, symptoms developed after infection by $F$. solani include leaf

\footnotetext{
*Correspondence: hhchen@mail.ncku.edu.tw

1 Department of Life Sciences, National Cheng Kung University,

701 Tainan, Taiwan

Full list of author information is available at the end of the article
}

spots, leaf blight, sheath rot, and root rot. The infection spread worldwide because of international trade and monoculture (Swett and Uchida 2015).

Fusarium solani with host specificity of Phalaenopsis has been found in Taiwan (Chung et al. 2011), Korea (Kim et al. 2002), Hawaii (Swett and Uchida 2015), and Australia (Laurence et al. 2016). Infection by $F$. solani causes a reduction of $20-30 \%$ yield every year in Taiwan (Su et al. 2010). To suppress the activity of Fusarium, 16 fungicides have been tested for growth inhibition of $F$. solani. However, although the fungicides showed effect to inhibit the mycelial growth of Fusarium (Su et al. 2014), none of them show significant effects on the reduction of the damage caused by $F$. solani in the greenhouse environment (Su et al. 2014), or after a long 
distance of shipment from country to country for several months (Liao et al. 2012). Instead of chemical treatment to reduce the infection rate of Fusarium on Phalaenopsis, development of resistant varieties with quantitative treat loci (QTLs) resistance to $F$. solani via breeding program will be an alternative solution. The resistance genes or QTLs to Fusarium spp. have been identified from Arabidopsis (Diener and Ausubel 2005), wheat (Buerstmayr et al. 2002) and pea (Coyne et al. 2015). However, so far no reports of Fusarium resistance QTL have been reported for orchids. Phenotyping symptoms after pathogen inoculation is the most important step for the identification of resistance genes or QTLs. A long duration of 4-6 weeks for disease progression after inoculation at the abbreviated stem of live Phalaenopsis plants have hampered the assessment. Even worse is that a large space is needed for examination enough plants for PhalaenopsisFusarium study (Su et al. 2012).

In this study, a modified method for assessment of the yellow leaf symptom infected by $F$. solani in detached leaf was established with reduced observation time of 6 days and much less space needed. In addition, the present study shows that similar trends of symptom development were observed between the infection of $F$. solani in the detached leaf and that in the abbreviated stem. Furthermore, symptom detection based on the detached leave method was stable and feasible for examining a large quantity of plants.

\section{Materials and methods}

\section{Sample collection}

Five Phalaenopsis cultivars including TAI_A2945, TAI A7403, TAI_A9168, TAI_A10040, TAI_A10746 from Taida Orchid Nursery were used in this research, to fit the real transportation situation, twenty mericlones cultivated were provided from orchid nursery, each individual was planted in 2.5-inch pots for 2-3 months which close to the true plant size for oversea production. Twenty individuals of each cultivar were used in each replicate and separated equally for Fusarium inoculation to detached leaf and abbreviated stem. For each tested tissue, 7 individuals were infected with Fusarium, and 3 individuals were inoculated with water as control. For each variety, a total of 60 plants were used, including 10 plants per tissue per variety were used in one replicate, and repeated 3 times independently. Three replicates in total were performed with oneweek interval among each replicate. Before the inoculation, the 2nd or 3rd leaf from the top was collected as a mature leaf based on the comparison of leaf length between 1st and 2nd leaf from the top. When the length of the 1st leaf shorter than half of the 2nd leaf, the 3rd leaf was represented as mature leaf. On the other hand, when the length of the 1st leaf longer than half of the 2nd leaf, the 2nd leaf was collected as matured leaf. The detached leaf was prepared from one mature leaf of each individual which cut around $8.5 \mathrm{~cm}$ from the apexes and then fixed the detached leaf in a $15 \mathrm{~cm}$ diameter dish. We kept all plant materials in a room with the constant temperature at $27^{\circ} \mathrm{C}$ for two days before the detached step to make sure all plants started with the same environment.

\section{Fusarium inoculation}

The procedures of inoculation for two organs were performed as previously described by Su et al. (2012). Briefly, seven wounds arranged in a circle was created before inoculation. The pathogenic fungi, Fusarium solani, was provided from Taiwan Agricultural Research Institute isolated from the orchid nursery in Taiwan. The spore suspension was diluted to $10^{3} \mathrm{pfu} / \mathrm{ml}$ for detached leaf inoculation and $10^{5} \mathrm{pfu} / \mathrm{ml}$ for the abbreviated stem inoculation. The seven wounds in a whorl was inoculated with $250 \mu \mathrm{l}$ inoculum of spore suspension at once and covered with micropore $(3 \mathrm{M})$ to maintain the liquid which was then removed 2 days after inoculation. The infected detached leaves, and plants infected at abbreviated stem were incubated in the climate control room (HiPoint, EH-1800) set at $27 \pm 1^{\circ} \mathrm{C}$ with $100 \%$ humidity. Photos of each infected detached samples were taken from the 2nd day to the 6th day after inoculation. For plants infected in abbreviated stem, photos were taken from the 2nd day after inoculation to the symptom rank 9 shown in any tested cultivars. The isolate of $F$ solani we used to inoculate plants was "TJP-2178-10", which showed the most severe symptom on 28 days post inoculation (dpi) (data not shown).

\section{Symptom ranking}

To distinguish the degree of pathogen resistance from all inoculated detached leaves or abbreviated stems, the disease severity level (DSL) was ranked each day after inoculation for 6 days (detached leaf) or for more than 3 weeks (abbreviated stem). Disease severity index (DSI) is a percentage calculated by the following formula. The number of individuals of each DSL level times each DSL, and make a summation of all different DSL. The sum is then divided by the score of the total levels of DSL times total number of individuals in the same variety (Chiang et al. 2017).

$$
D S I(\%)=\frac{\sum(D S L \times \text { number of individuals in this level of variety })}{\text { Tatal level of DSL times total number of individuals in the same variety }}
$$


For instance, we have 7 individuals in total 11 ranks of DSL, 3 of them ranked as DSL 3 and the rest of 4 ranked as DSL 4 , then the DSI here will be calculated as following: $(3 * 3+4 * 4) /(11 * 7)^{*} 100 \%=32.4 \%$.

\section{Results}

Determination of DSL ranks for the infected detached leaf and infected plant at abbreviated stem

A pre-examination was performed to distinguish the different ranks of DSL by inoculating Fusarium on the detached leaf or at the abbreviated stem. Variety TAI A10746 was selected to show the scale of DSL, except the rank 10 of detached leaf (variety $\mathrm{CH} 151$, Fig. 1).
The DSL was ranked as followed: Level 0: no symptom; level 1: number of black holes less than 3; level 2: number of black holes between 4 and 7; level 3: black holes connect together; level 4: black lesion spreads outside of the wound; level 5: less than 3 holes filled by the hypha from F. solani; level 6: 4 to 7 holes filled by hypha from F. solani; level 7: the hypha mixed together; level 8: yellow symptom emerges; level 9: yellow symptom diffuses to an entire detached leaf or abbreviated stem; level 10: the red spores show (Figs. 1 and 2). These DSL levels were applied to the photos taken from all five varieties, and three replicates for calculating the DSI of different tissues.
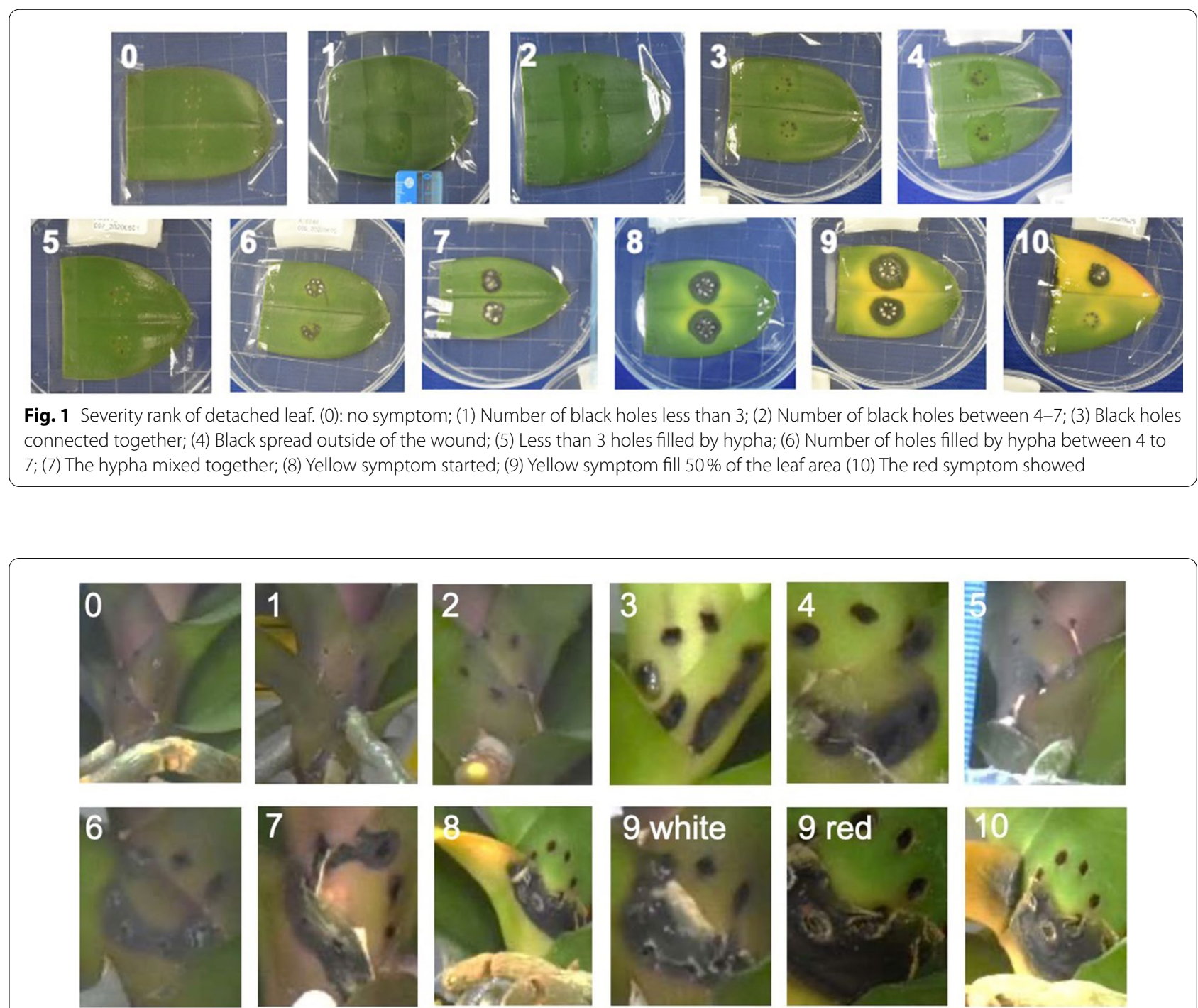

Fig. 2 Severity rank of abbreviated stem. (0): No black holes; (1) Number of black holes less than 3; (2)Number of black holes between 4-7; (3) Black holes connected together; (4) Black spread outside of the wound; (5) Less than 3 holes filled by hypha; (6) Number of holes filled by hypha between 4 to 7; (7) A region of white area showed; (8) Yellow symptom showed; (9) White / Red spots appeared; (10) Yellow leaf broke 
Similar trends of symptom development from detached leaf and abbreviated stem inoculated with $F$. solani

According to previous test, cultivar TAI_A7403 and TAI_A10040 were highly tolerant and susceptible to $F$. solani infection. We first checked whether the symptoms on detached leaf and abbreviated stem would be similar after inoculated with $F$. solani. First of all, the symptoms of tolerant and susceptive cultivars that developed on the detached leaf and abbreviated stem were shown. There were no symptoms in the detached leaf and abbreviated stem of variety TAI_A7403 (Fig. 3a, b, e, f). In contrast, strong yellow leaf symptoms in both the detached leaf and the abbreviated stem were revealed for variety TAI A10040 (Fig. 3c, d, g, h). Second, similar disease severity index patterns were recorded among five tested varieties between detached leaf and abbreviated stem (Fig. 4). Highly positive correlations were detected between the infected detached leaf and the infected plants at abbreviated stem among replicates, as the $\mathrm{R}$ square was 0.96 , 0.91 , and 0.95 for replicate $1,2,3$, respectively, and p-value lower than 0.05 of all replicates (Table 1).

\section{Stability of detection and reduction of the observation period by infection in the detached leaf}

The stability of DSI among replicates developed on each organ is crucial for the evaluation of the reliability of the inoculation technique. The DSIs for both infected detached leaf and infected plants at abbreviated stem showed significant highly correlated among all replicates (Table 2), suggesting that tested varieties showed consistent yellow leaf symptoms from these organs, and both infections at detached leaf and at abbreviated stem are reliable inoculation techniques. The detached leaf has the highest correlation coefficient 0.96 between replicate 2 and 3 , and the lowest correlation coefficient 0.84 between replicate 1 and 2 with the p-value 0.0081 and 0.0771 respectively. In contrast, the correlation among replicates from the abbreviated stem was higher than 0.9 with p-value 0.0292 (replicate 1 vs. replicate 2), 0.0132 (replicate 1 vs. replicate 3), and 0.0299 (replicate 2 vs. replicate 3) (Table 2). In addition, symptom observation time is also an essential point to be considered before screening a large number of samples or varieties. In this study, it took about 20 days for abbreviated stem (ranged from 21 to 32 days), while it took less than one week (6 days) for the detached leaf to differentiate the DSI among orchid varieties after infection with $F$. solani.

\section{Discussion}

Infection of detached leaf is a better approach for assessment of DSI upon F. solani inoculation

A good pathogeny assessment technology may consider the following features: repeatability, sensitivity, and observation time. Different techniques of Fusarium inoculation has been tested in maize ear to find out the best way for evaluating a large number of maize genotypes (Clements et al. 2003). In our study, high correlations detected among all replicates from both organs (Table 2) indicating the trusted repeatability. In addition, the severity pattern from two organs was highly correlated among replicates (Table 1). A similar severity pattern after Fusarium inoculation between organs is observed from ear and silk in maize as well (Reid et al. 2002). This finding indicates that the severity rank obtained from

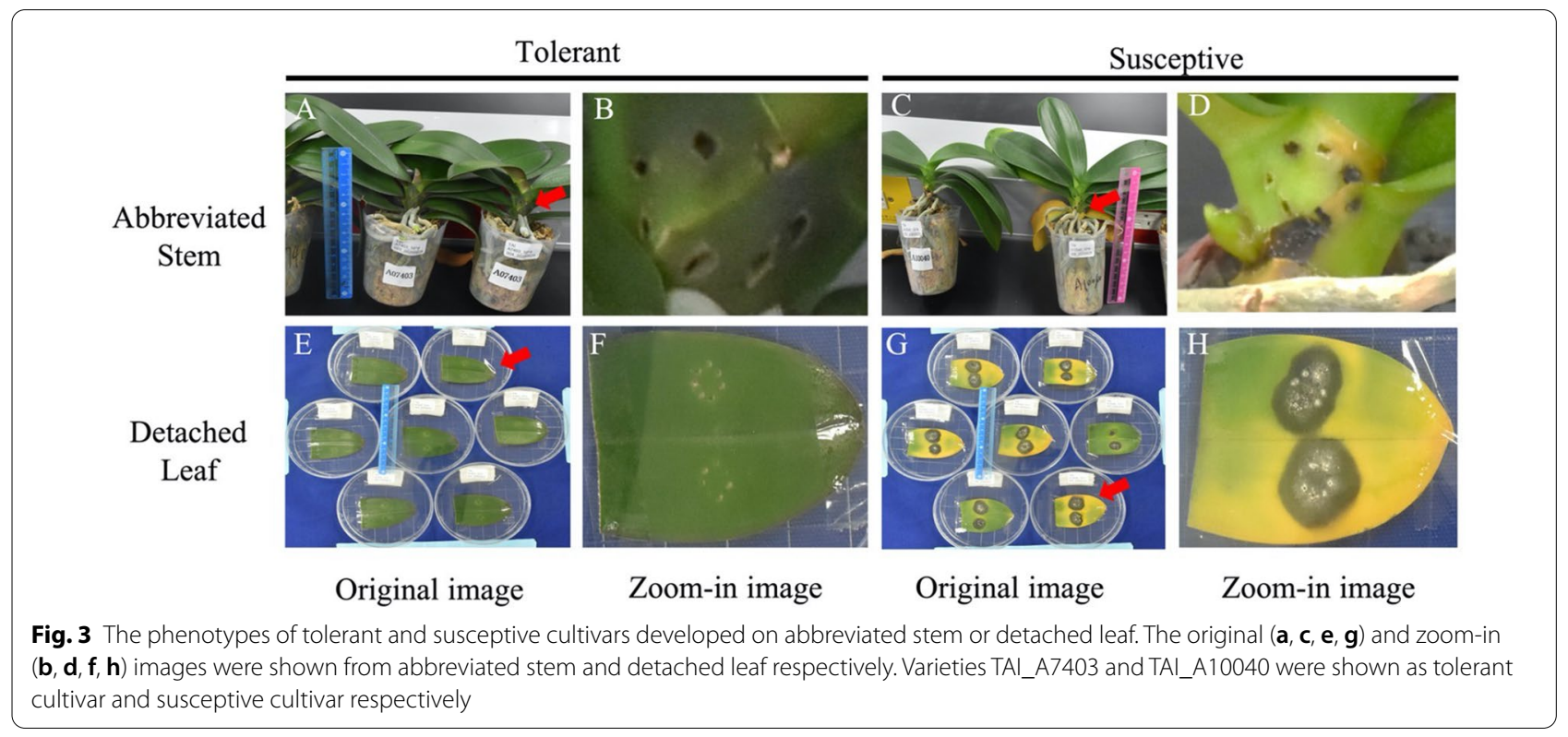




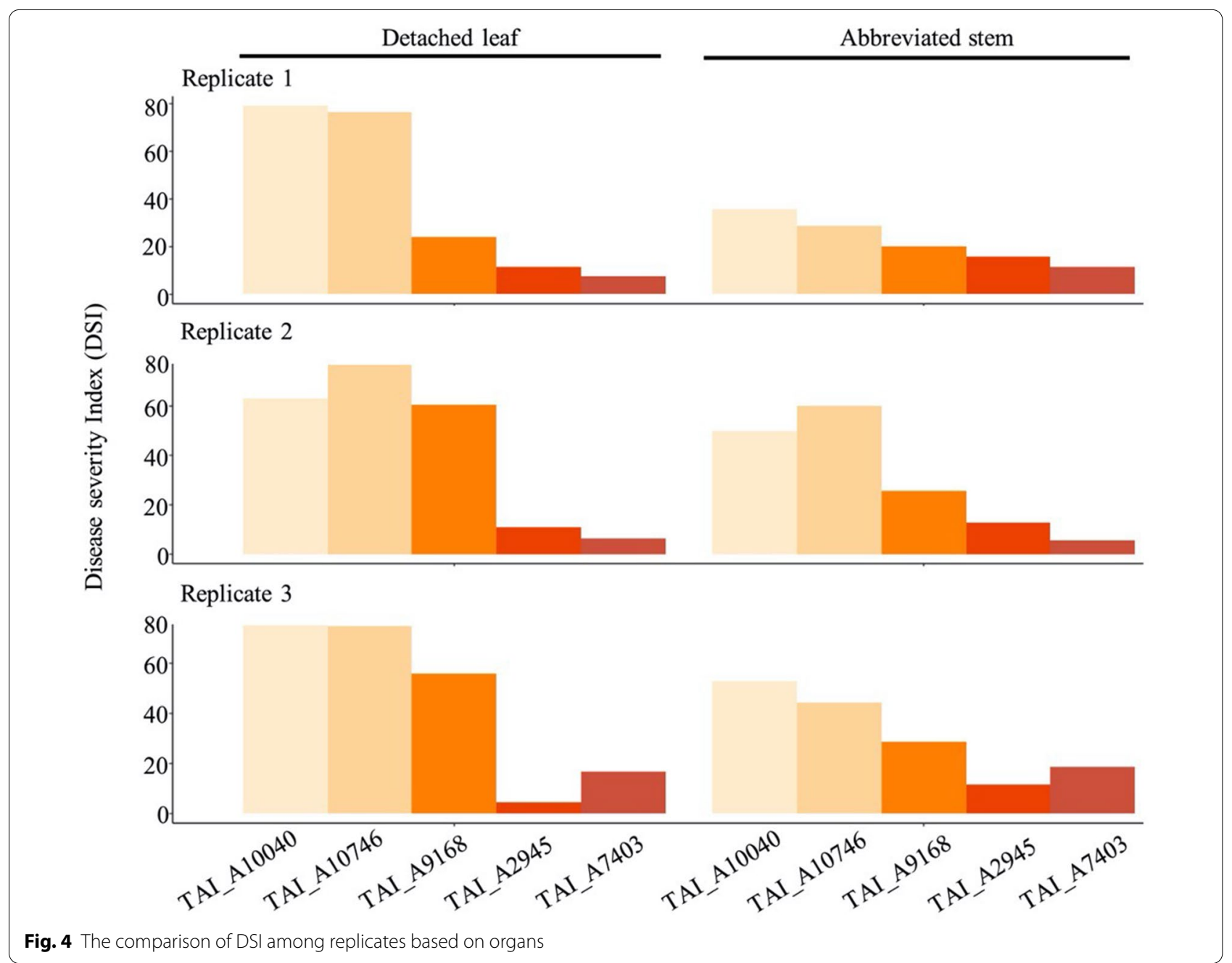

Table 1 The correlation of each replicate between abbreviated stem and detached leaf

\begin{tabular}{llll}
\hline & Replicate 1 & Replicate 2 & Replicate 3 \\
\hline$r$ & 0.96 & 0.91 & 0.95 \\
$p$ value & 0.0099 & 0.0309 & 0.0125 \\
\hline
\end{tabular}

Table 2 The correlation between each replicate of detached leaf and abbreviated stem, above the diagonal indicates the correlation coefficient $(r)$ and below the diagonal showed p-value

\begin{tabular}{llll}
\hline \multicolumn{4}{c}{ Detached Leaf / Abbreviated Stem } \\
\hline Replicate & I & ॥ & II \\
I & & $0.84 / 0.92$ & $0.89 / 0.95$ \\
II & $0.0771 / 0.0292$ & & $0.96 / 0.91$ \\
III & $0.0409 / 0.0132$ & $0.0081 / 0.0299$ & \\
\hline
\end{tabular}

the detached leaf well represents the rank detected from the abbreviated stem. Furthermore, the detached leaf showed a wider DSI range among tested varieties than the abbreviated stem, displaying the stronger sensitivity from detached leaf than abbreviated stem. Regarding the observation time, more than 20 days were needed for symptom development after inoculation in the abbreviated stem, similar to a previous study that took 4 weeks for symptom detection in orchids (Su et al. 2012). In contrast, 6 days after inoculation with $F$. solani was enough to distinguish the ranges of symptoms from detached leaf, indicating one-fourth time of inoculation in the abbreviated stem. Last but not least, the space requirement is also an important issue. It was less spacy for the detached leaf as compared to that for the abbreviated stem. A dish with $15 \mathrm{~cm}$ diameter and $2 \mathrm{~cm}$ height are enough for one sample of the detached leaf, but for the abbreviated stem, a living plant in a 2.5 -inch pot is required. From all above 
mentioned advantages, inoculation in the detached leaf is reliable, more sensitive, shorter time consuming and less experiment space needed than the inoculation in the abbreviated stem, which could be the better solution for Fusarium assessment in Phalaenopsis orchids.

\section{Examining the biochemical defense of Phalaenopsis-Fusarium interaction}

For the defense of the invasion from pathogens, plants develop both structural and biochemical mechanisms to protect themselves. When Fusarium spp. infects plants, it stars to destroy the structural defense of cell wall, and then override the plant biochemical defense by producing host-specific mycotoxins (Perincherry et al. 2019). In this study, the first structural defense was avoid since plants were punctured and round wounds were created, which brought the Fusarium into the interior part of the plant cells and examined the biochemical defense. In this way, we tested the pathogen-specific host defense directly, and will allow the identification of important factors involved in the defense mechanism for Fusarium infection in the future.

\section{Conclusions}

Phalaenopsis orchids play the most significant role in flower exportation in Taiwan, but the orchid flower yield has been reduced by the yellow leaf disease caused by Fusarium spp. Phenotyping of Fusarium symptom is the first step and the most necessary information for further studies such as genome-wide association study. The detached leaf, a better tissue than the original inoculation tissue, the abbreviated stem for inoculation of Fusarium isolate "TJP-2178-10" was provided in this study and proved to be with similar distinguish ability, good stability, while shorter time-consuming and lesser space needed. This solution allows a better phenotyping approach and fast detection of yellow leaf disease associated with Fusarium for a large number of Phalaenopsis samples.

\section{Abbreviations}

DSI: Disease severity index; DSL: Disease severity level; QTLs: Quantitative treat loci.

\section{Acknowledgements}

We thank Lan-Yi Chien (Plant Pathology Division, Taiwan Agricultural Research Institute Council of Agriculture) for technical assistance of Fusarium infection.

\section{Authors' contributions}

SYC conceived the original screening and research plans; YJW performed the experiment together; TFH and JFS supervised the experiments; LYC assist the infection of Fusarium process; WCS participated in the discussion after the experiment; YHL and PCL provided plant materials for pre-test and internal controls for different lots of experiments, participated the experiment design and discussion after analysis; WHC participated the design of experiment and the analysis after the experiment; $\mathrm{HHC}$ conceived the project and wrote the article with contributions of all the authors, completed the writing, and serve as the author responsible for contact and ensures communication. All authors read and approved the finalmanuscript.

\section{Funding}

This work was supported by Ministry of Science and Technology, Taiwan, Grant No.: MOST 108-2313-B-006-MY3.

\section{Availability of data and materials}

Not applicable.

\section{Declarations}

Ethics approval and consent to participate

Not applicable.

Consent for publication

Not applicable.

\section{Competing interests}

The authors declare that they have no competing interests.

\section{Author details}

${ }^{1}$ Department of Life Sciences, National Cheng Kung University, 701 Tainan, Taiwan. ${ }^{2}$ Plant Pathology Division, Taiwan Agricultural Research Institute Council of Agriculture, 413008 Taichung, Taiwan. ${ }^{3}$ Department of Plant Pathology and Microbiology, National Taiwan University, 10617 Taipei, Taiwan. ${ }^{4}$ Taida Horticultural Co., Ltd, Da Tsun County, 31306 Chang Hwa, Taiwan.

${ }^{5}$ Orchid Research and Development Center, National Cheng Kung University, 701 Tainan, Taiwan.

Received: 9 November 2020 Accepted: 16 March 2021

Published online: 31 March 2021

\section{References}

Buerstmayr H, Lemmens M, Hartl L, Doldi L, Steiner B, Stierschneider M, Ruckenbauer P (2002) Molecular mapping of QTLs for fusarium head blight resistance in spring wheat. I. Resistance to fungal spread (type ii resistance). Theor Appl Genet 104:84-91

Chiang K, Liu H, Bock C (2017) A discussion on disease severity index values. Part I: warning on inherent errors and suggestions to maximise accuracy. Ann Appl Biol 171:139-154

Chung W, Chen L, Huang J, Huang H, Chung W (2011) A new'forma specialis' of Fusarium solani causing leaf yellowing of phalaenopsis. Plant Pathol 60:244-252

Clements M, Kleinschmidt C, Maragos C, Pataky J, White D (2003) Evaluation of inoculation techniques for fusarium ear rot and fumonisin contamination of corn. Plant Dis 87:147-153

Coyne CJ, Pilet-Nayel ML, McGee RJ, Porter LD, Smýkal P, Grünwald NJ (2015) Identification of QTL controlling high levels of partial resistance to Fusarium solani f. sp. pisi in pea. Plant Breed 134:446-453

Diener AC, Ausubel FM (2005) Resistance to Fusarium Oxysporum 1, a dominant arabidopsis disease-resistance gene, is not race specific. Genetics 171:305-321

Kim WG, Lee BD, Kim WS, Cho WD (2002) Root rot of moth orchid caused by Fusarium spp. Plant Pathol J 18:225-227

Laurence M, Howard C, Summerell B, Liew E (2016) Identification of Fusarium solani f. Sp. phalaenopsis in australia. Australas Plant Dis Notes 11:15

Liao G, Hsieh TF, Chen HR (2012) Effect of chemical treatment of prevention of fusarium yellow of Phalaenopsis seedlings under simulated conditions of shipment. J Taiwan Agri Res 61:124-131

Perincherry L, Lalak-Kańczugowska J, Stępień $Ł$ (2019) Fusarium-produced mycotoxins in plant-pathogen interactions. Toxins 11:664

Reid L, Woldemariam T, Zhu X, Stewart D, Schaafsma A (2002) Effect of inoculation time and point of entry on disease severity in Fusarium 
graminearum, Fusarium verticillioides, or Fusarium subglutinans inoculated maize ears. Can J Plant Path 24:162-167

Su JF, Lee Y, Chen C, Hsieh TF, Huang J (2010) Sheath and root rot of Phalaenopsis caused by Fusarium solani. Acta Hortic 878:389-394. https://doi. org/10.17660/ActaHortic.2010.878.49

Su JF, Lee Y, Chen C, Huang J, Hsieh TF (2012) Field surveys for Fusarium diseases of Phalaenopsis in Taiwan. Plant Pathology Bull 21:115-130

Su JF, Lee YC, Chien LY, Hsieh TF (2014) Evaluation of fungicides for growth inhibitions of Phalaenopsis Fusarium pathogen. J Taiwan Agri Res 64:32-44
Swett C, Uchida J (2015) Characterization of Fusarium diseases on commercially grown orchids in Hawaii. Plant Pathol 64:648-654

\section{Publisher's note}

Springer Nature remains neutral with regard to jurisdictional claims in published maps and institutional affiliations.

\section{Submit your manuscript to a SpringerOpen ${ }^{\circ}$ journal and benefit from:}

- Convenient online submission

- Rigorous peer review

- Open access: articles freely available online

- High visibility within the field

- Retaining the copyright to your article

Submit your next manuscript at $\boldsymbol{\nabla}$ springeropen.com 